\title{
OpenCell
}

\section{Status and plans}

\section{Alan Garny}

alan.garny@dpag.ox.ac.uk 


\section{Various Versions of OpenCeLl}

- Several versions of OpenCell (formerly known as PCEnv) over the past few years. Some key features include:

- PCEnv 0.4: graphical rendering of mathematics, units, etc., and C code export; 


\section{Various Versions of OPEnCELL}

- Se

Piysiome GellML Environment

In as $\mathrm{PC}$ V.ew change tree : inc$$
\text { ( }
$$

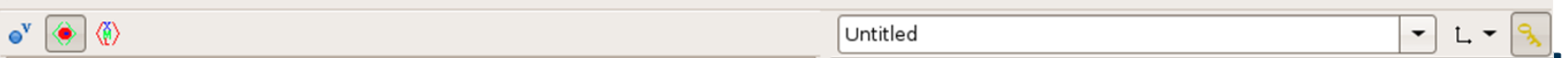
- cheng_model_2000 Type Mathematics 12 =Equation

$\nabla \times$ Mathematics 13 =Equation

$\nabla \times-$ Mathematics 14

= Equation

$\nabla+\cdots$ Mathematics 15

=Equation $\nabla_{घ^{c} \vee}$

$D Q^{v} V \quad 0.093$

$D Q^{\mathrm{v}}$ mass

0.005

$D \theta^{v} F_{-}$total

$D_{0} v_{\text {time }}$

Equation $T_{-} f=$ piecewise(case df_eff_dt $>=0.0$ \{units="dimensionless" $\}$ then $T_{-}$

$\mathrm{T}_{-} \mathrm{f}=\left\{\begin{array}{c}\mathrm{T}_{-} \mathrm{f} 1 \times L^{2.0}+\mathrm{T}_{-} \mathrm{f} 2 \times \mathrm{f}_{-} \text {env if df_eff_dt } \geq 0.0 \\ \frac{\mathrm{T}_{-} \mathrm{f} 3+\mathrm{T}_{-} \mathrm{f} 4 \times \mathrm{Af}}{L} \text { otherwise }\end{array}\right.$

first_order_rate_constant

kilogram

newton

millisecond

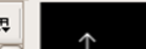
tures

units, 


\section{Various Versions of OpenCeLl}

- Several versions of OpenCell (formerly known as PCEnv) over the past few years. Some key features include:

- PCEnv 0.4: graphical rendering of mathematics, units, etc., and C code export;

- PCEnv 0.5: visual validator, experimental DAE solver; 


\title{
Various Versions of OpenCell
}

C Plivsiome GellML Environment

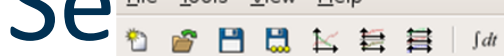

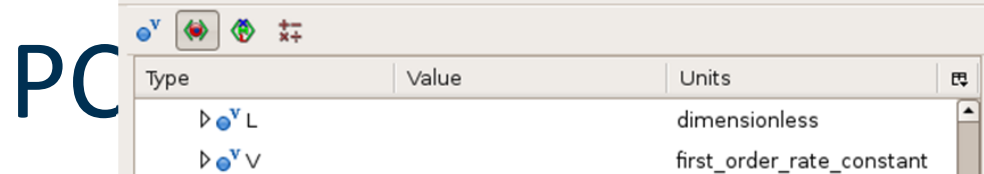

1 Waming: Expected all arguments to MathML apply to have the same units

$\triangle$ Waming: Expected arguments to MathML apply to have dimensionless units

vn as

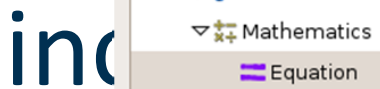

1 Waming: Expected all arguments to MathML apply to have the same units

$\triangle$ Waming: MathML equals element has inconsistent units between the sides

Error: Connection of two variables which have dimensionally inconsistent units

X Error: Mapping variable_l has public interface of in but variable_2 also has public interface of in

Xrror: connection of two variables which have dimensionally inconsistent units

X Error: Mapping variable_1 has public interface of in but variable_2 also has public interface of in

Error: Connection of two variables which have dimensionally inconsistent units

ロ

$D \square^{c} A f$

$D_{\square^{C}} F_{-} C E$

$D_{\square^{C} F_{-} \text {total }}$

$D \square^{c} L_{-}$eff

$D_{\Xi^{C} S} \mathrm{~S}$

$D a^{c} Y$

Equation $\mathrm{FV}=$ piecewise (case $\vee<=0.0$ \{units="dimensionless" $\}$ then $(\mathrm{V}$ ma:

X Error: Mapping variable_1 has public interface of in but variable_2 also has public interface of in

r;

E Eror: Connection of two variables which have dimensionally inconsistent units

X Error: Mapping variable_1 has public interface of in but variable_2 also has public interface of in

X Error: Connection of two variables which have dimensionally inconsistent units

Х Error: Mapping variable_1 has public interface of in but variable_2 also has public interface of in

$\mathrm{FV}=\left\{\begin{array}{c}\frac{\mathrm{V}_{-} \mathrm{max}-V}{\mathrm{v}_{-} \mathrm{max}+(\mathrm{cv} 0+\mathrm{cv} 1 \times L) \times V} \text { if } V \leq 0.0 \\ \frac{\mathrm{bv}-\left(\left(\mathrm{av} 0+\mathrm{av} 1 \times L+\mathrm{av} 2 \times L^{2.0}\right) \times V\right)}{\mathrm{bv}+V} \text { otherwise }\end{array}\right.$

X Eror: connection of two variables which have dimensionally inconsistent units

X Error: Mapping variable__ has public interface of in but variable_2 also has public interface of in

\author{
units,
} tures 


\section{Various Versions of OpenCeLl}

- Several versions of OpenCell (formerly known as PCEnv) over the past few years. Some key features include:

- PCEnv 0.4: graphical rendering of mathematics, units, etc., and $C$ code export;

- PCEnv 0.5: visual validator, experimental DAE solver;

- PCEnv 0.6: MATLAB and Python code export in addition to $C$ (incl. DAE models), as well as Mac OS $X$ support; 


\section{Various Versions of OpenCell}

- Sego

hysiome CellML Environment File Tools View Help

Physiome CellML Environment

口 * $\bigcirc$ Thu $16: 34: 42$ cmiss Q

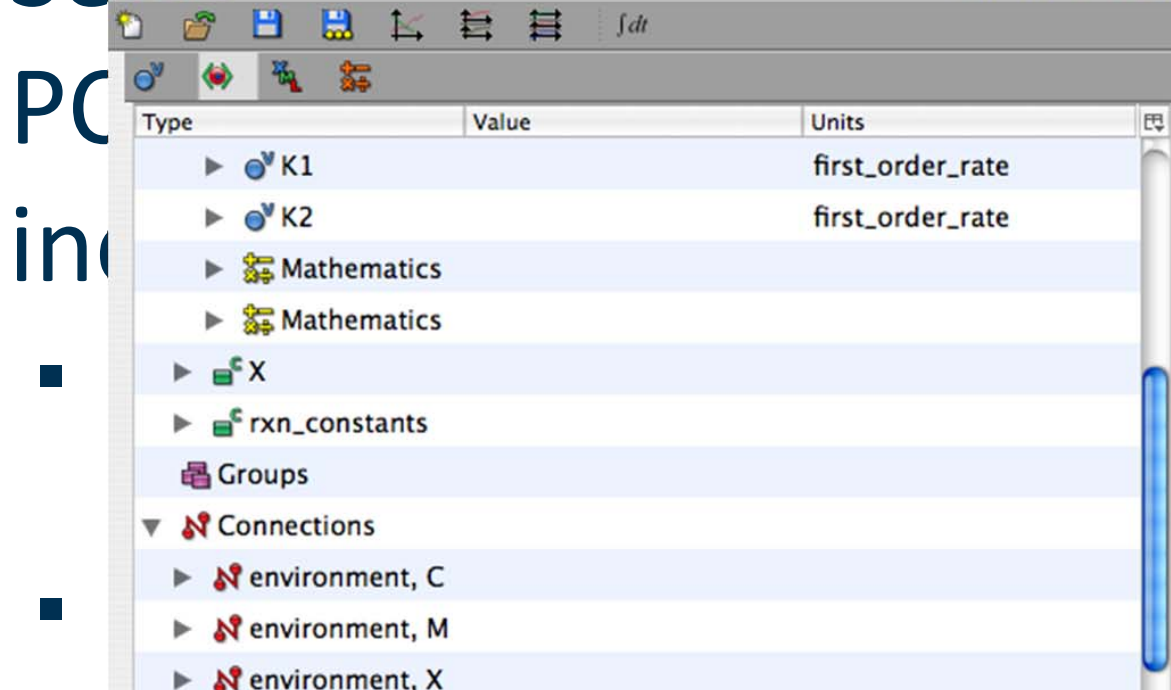

- Nenvironment, $\mathrm{X}$

- $\mathbb{N}^{\mathrm{C}} \mathrm{C}, \mathrm{rxn}_{-}$consta...

- Sic, $x$

- S M, rxn_consta..

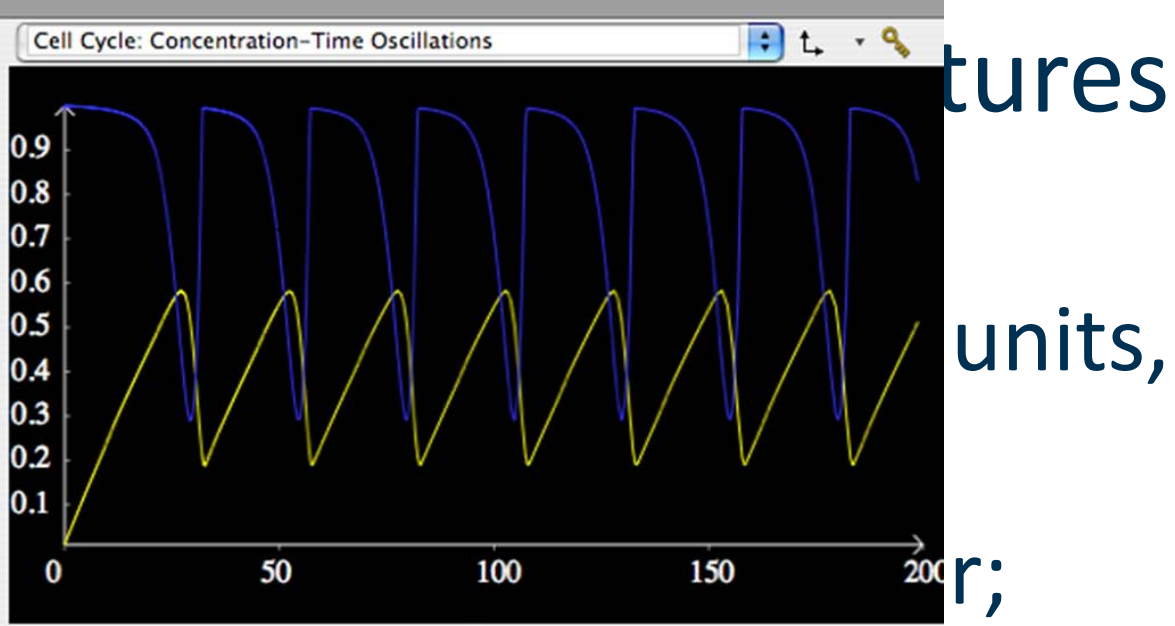

(3) dition

C

rxn_constants

$\mathrm{kd}$ (first_order_rate) $\leftarrow \mathrm{kd}$ (first_order_rate)

$\mathrm{Kd}$ (micromolar) $\leftarrow \quad \mathrm{Kd}$ (micromolar)

vi (second_order_rate) $\leftarrow$ vi (second_order_rate)

$\mathrm{vd}$ (second_order_rate) $\leftarrow$ vd (second_order_rate)

$\mathrm{C}$ (micromolar) $\rightarrow \quad \mathrm{C}$ (micromolar)

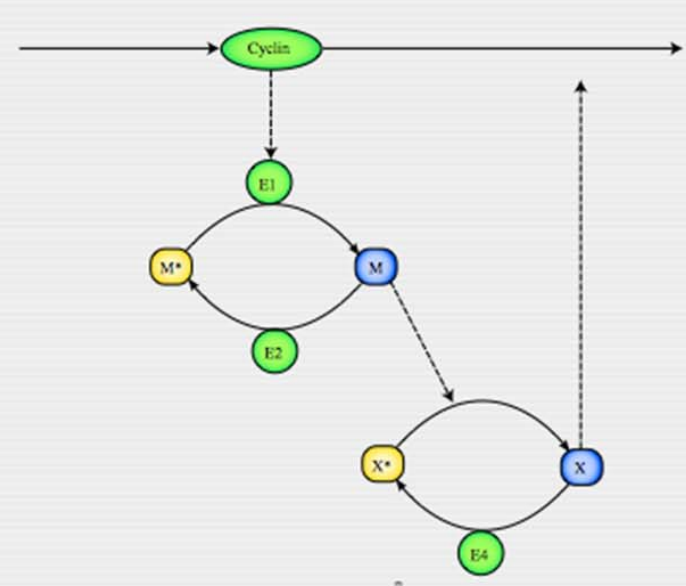

$\mathrm{t}$; 


\section{Various Versions of OpenCeLl}

- Several versions of OpenCell (formerly known as PCEnv) over the past few years. Some key features include:

- PCEnv 0.4: graphical rendering of mathematics, units, etc., and $C$ code export;

- PCEnv 0.5: visual validator, experimental DAE solver;

- PCEnv 0.6: MATLAB and Python code export in addition to $C$ (incl. DAE models), as well as Mac OS $X$ support;

- OpenCell 0.7: support for drag and drop editing of connections and graph traces, multiline math input, experimental Fortran 77 code export; and 


\section{Various Versions of OpenCeLl}

- Several versions of OpenCell (formerly known as PCEnv) over the past few years. Some key features include:

- PCEnv 0.4: graphical rendering of mathematics, units, etc., and $C$ code export;

- PCEnv 0.5: visual validator, experimental DAE solver;

- PCEnv 0.6: MATLAB and Python code export in addition to $C$ (incl. DAE models), as well as Mac OS $X$ support;

- OpenCell 0.7: support for drag and drop editing of connections and graph traces, multiline math input, experimental Fortran 77 code export; and

- OpenCell 0.8RC1: support for IDA as an integrator, copy and paste of parts of models from the tree views. 


\section{Editing IN OPENCELL}

- Editing of CellML files can be done using:

- The initial conditions/constants view;

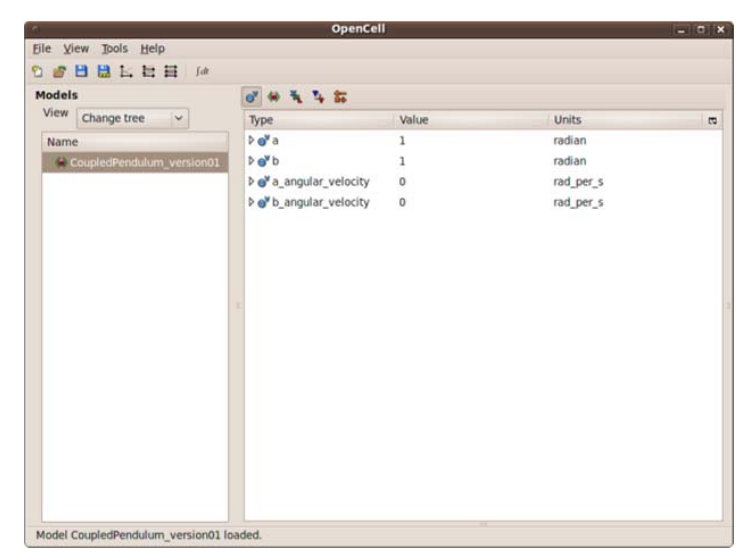




\section{EDITING IN OPENCELL}

- Editing of CellML files can be done using:

- The initial conditions/constants view;

- The complete model structure view;
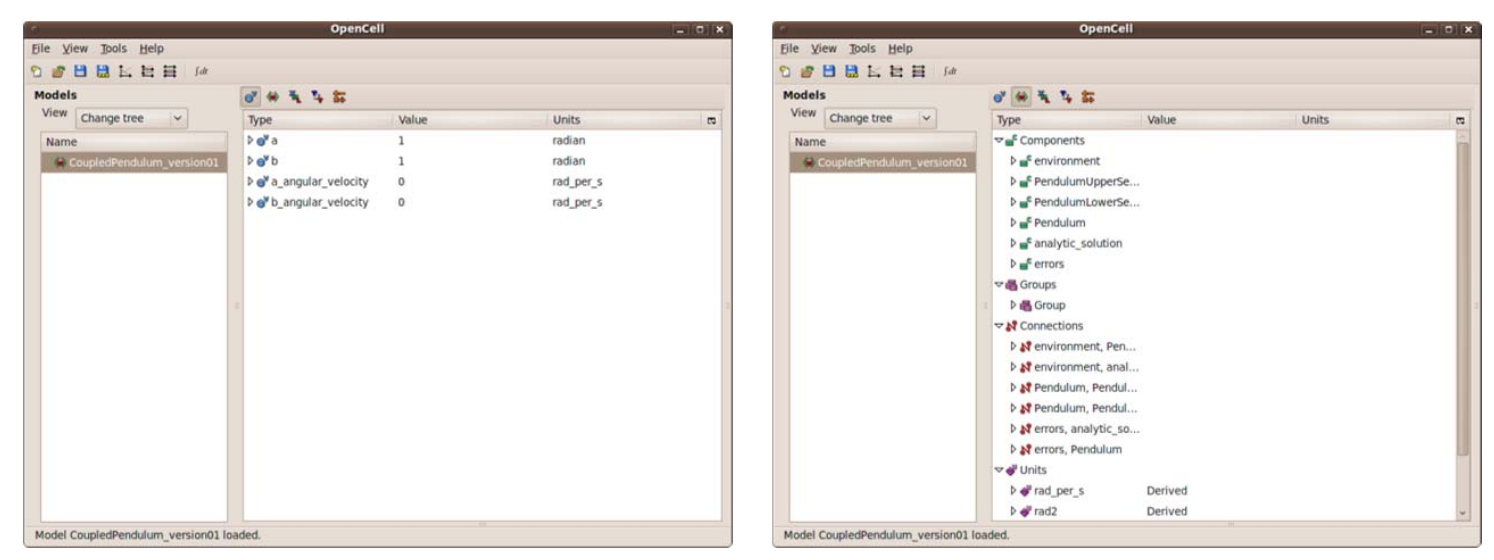


\section{EdITING IN OPENCELL}

- Editing of CellML files can be done using:

- The initial conditions/constants view;

- The complete model structure view;

- The XML view; or
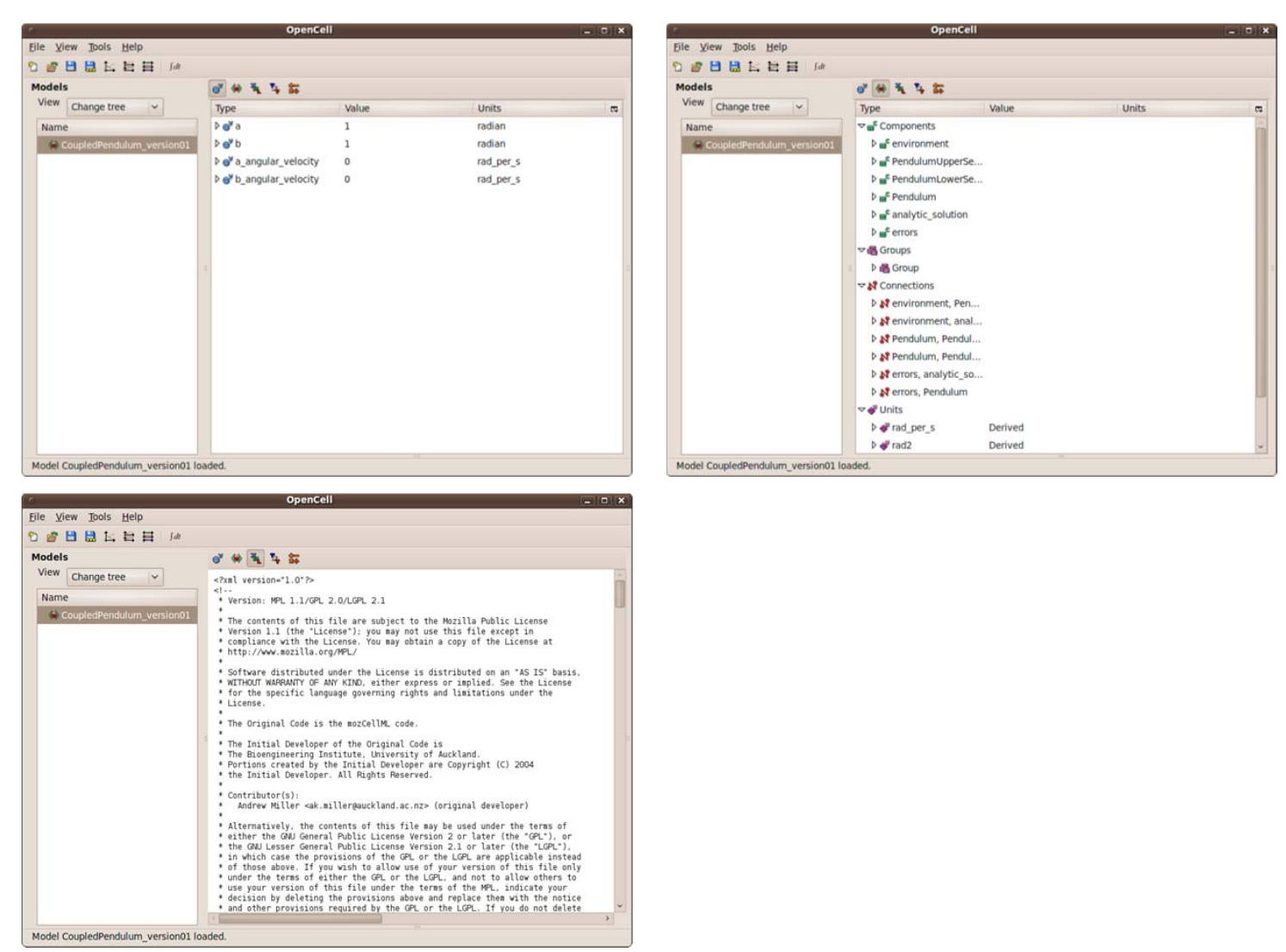


\section{EDITING IN OPENCELL}

- Editing of CellML files can be done using:

- The initial conditions/constants view;

- The complete model structure view;

- The XML view; or

- The equations view.
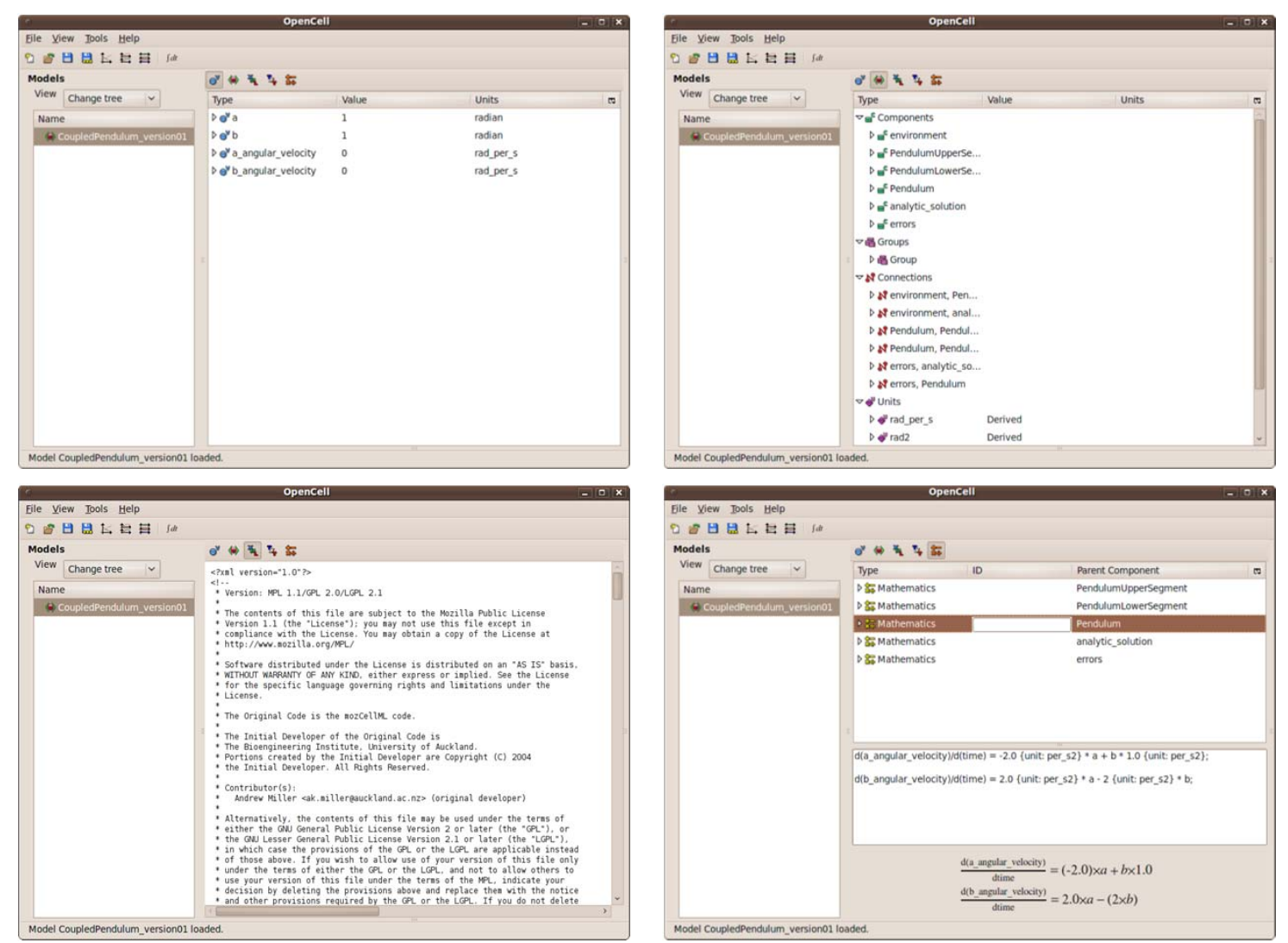


\section{EDITING IN OPENCELL}

- Editing of CellML files can be done using:

- The initial conditions/constants view;

- The complete model structure view;

- The XML view; or

- The equations view.

- However, this may not always be the fastest and/or most obvious way to edit a CellML file.

- Another possible approach is that of COR which relies on a proprietary language.

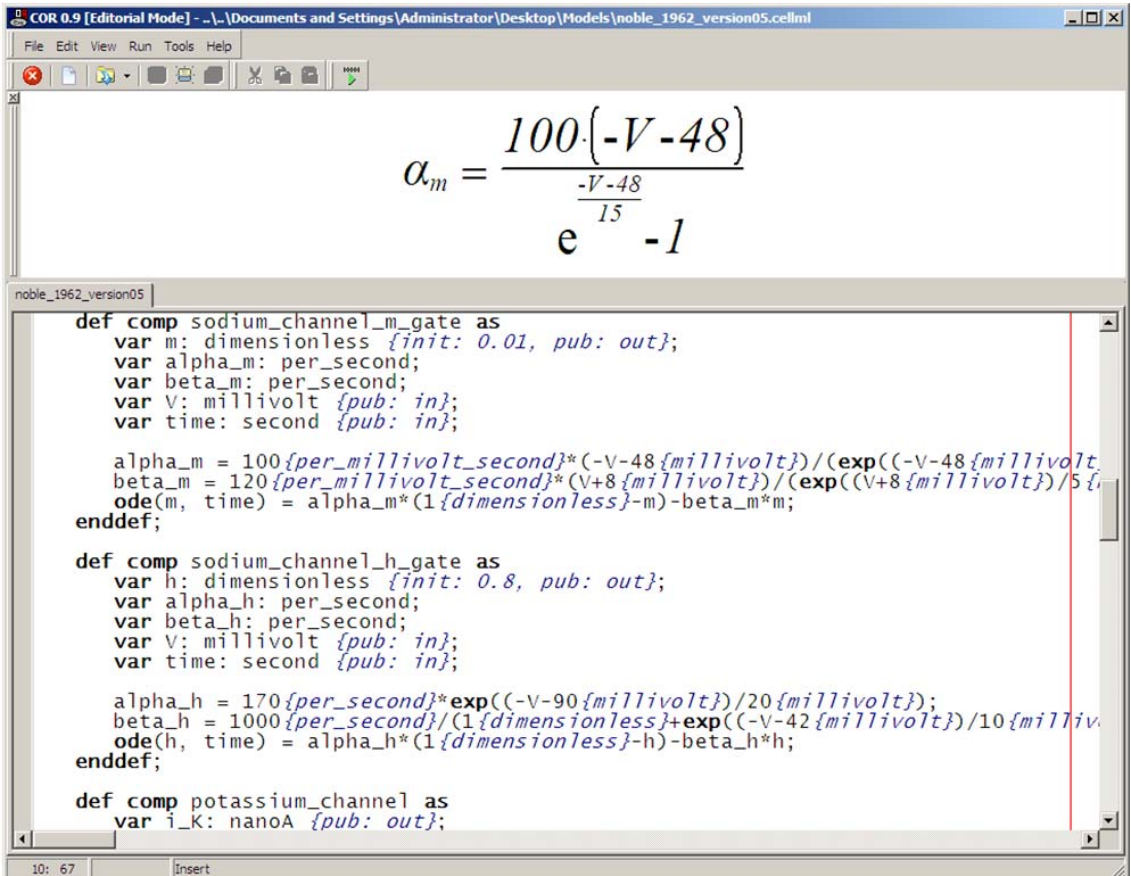




\section{COR-LIKE VIEW IN OPENCELL}

- Not $100 \%$ compatible with COR, but CellML 1.1 capable.

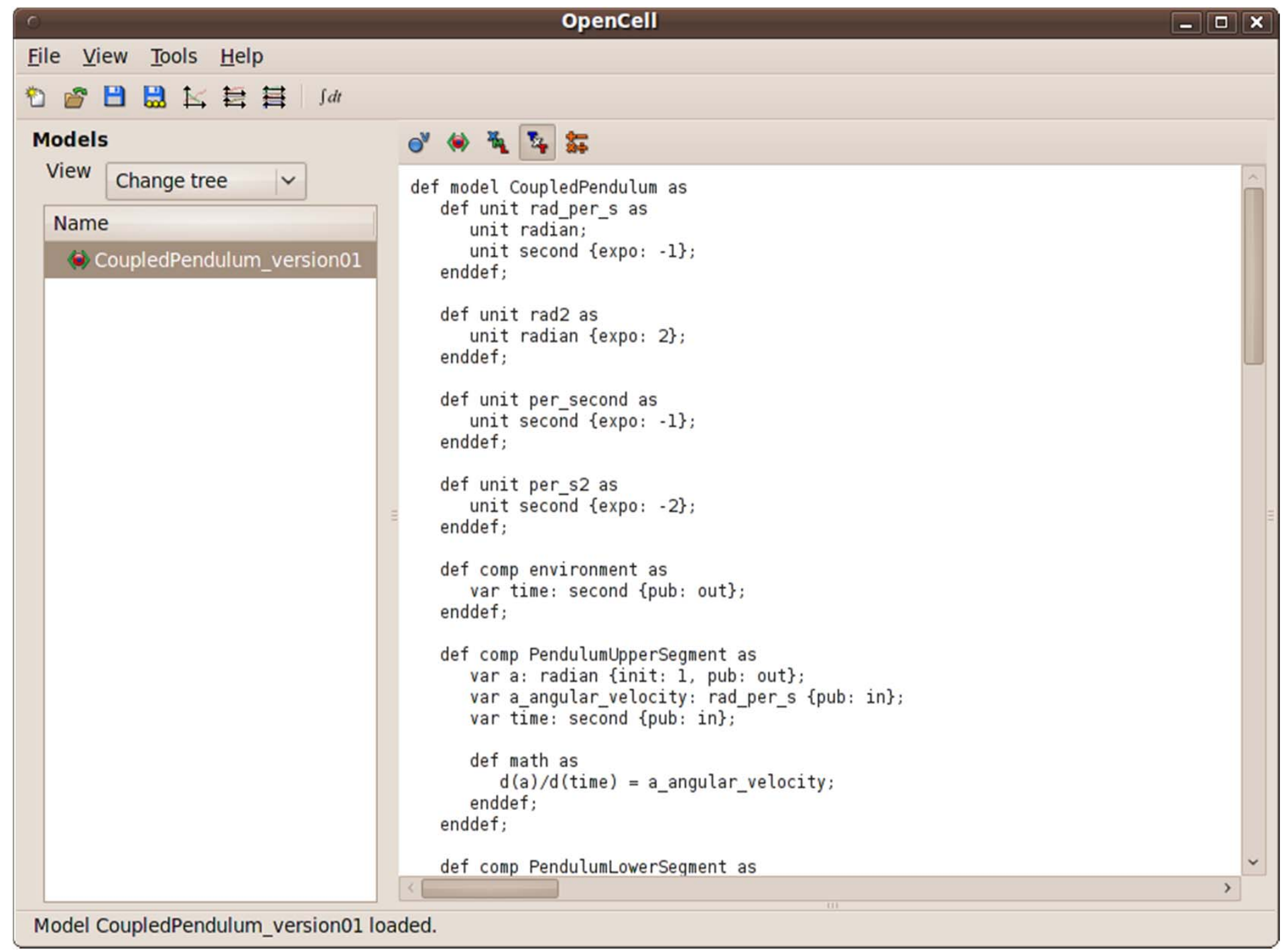




\section{Plan for a New OpenCell}

- OpenCell currently relies on the Mozilla XULRunner Framework, making it difficult to develop OpenCell further.

- New OpenCell to be developed using Qt/C++.

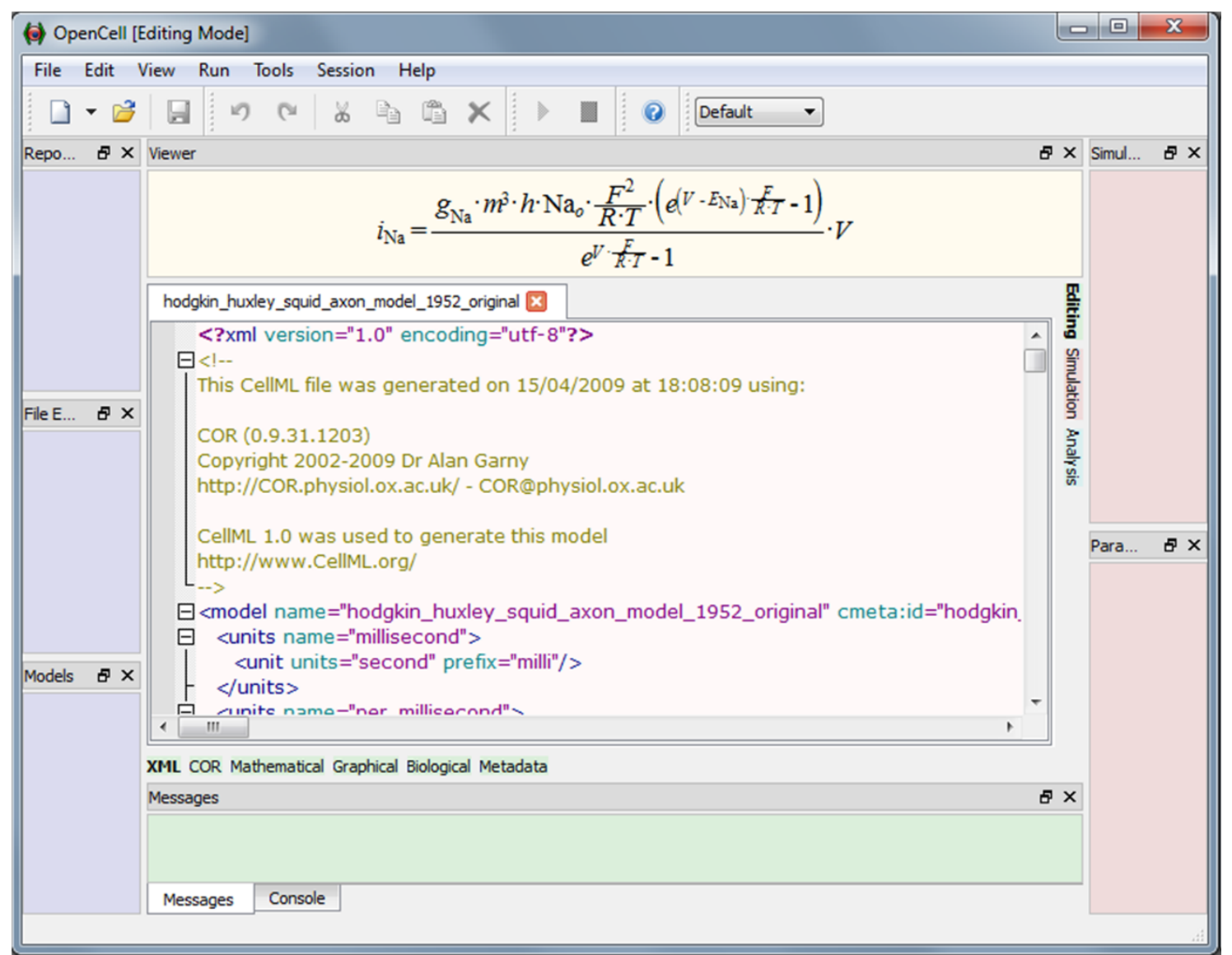




\section{Plan for a New OpenCell}

- OpenCell currently relies on the Mozilla XULRunner Framework, making it difficult to develop OpenCell further.

- New OpenCell to be developed using Qt/C++.

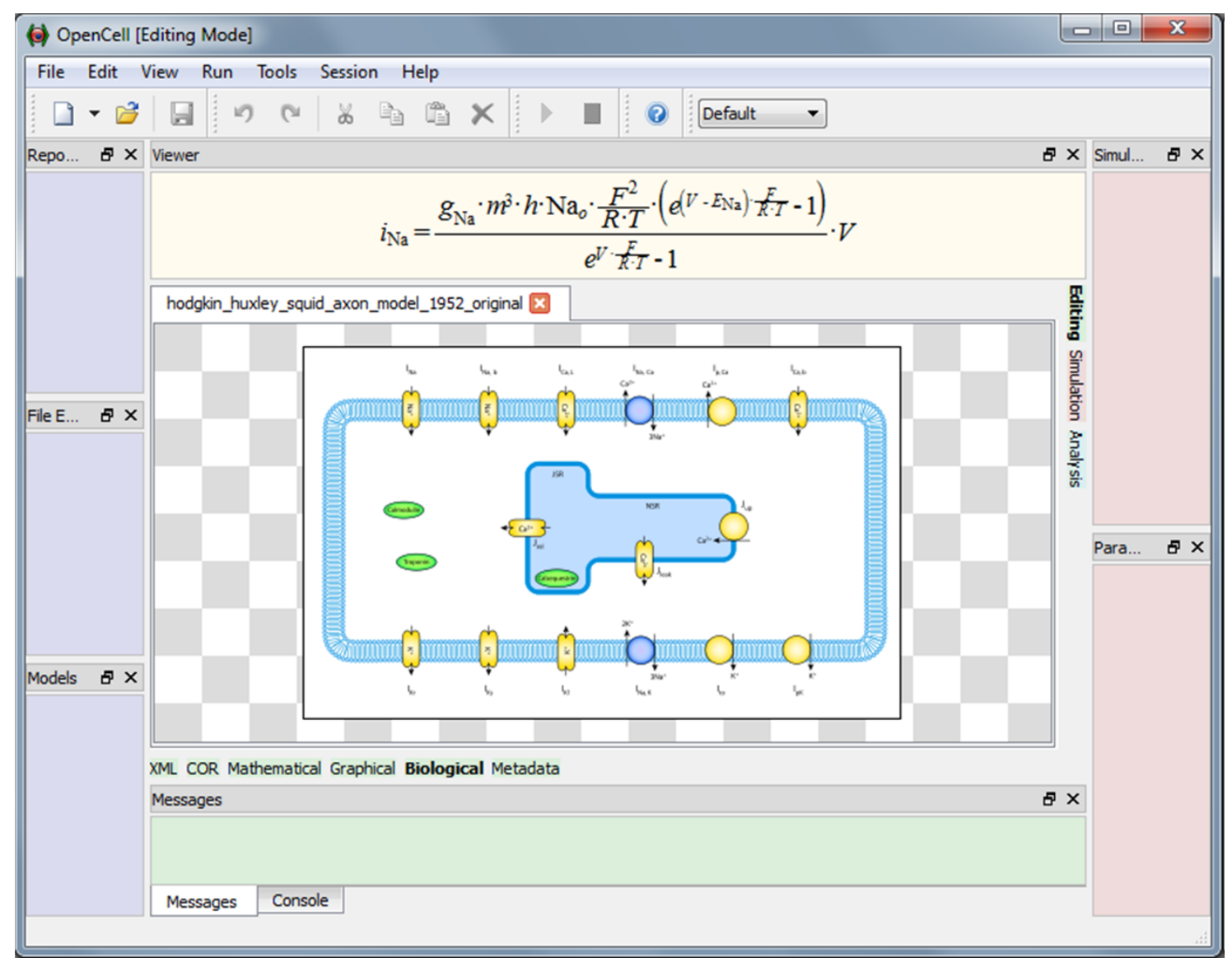




\section{Plan for a New OpenCell}

- OpenCell currently relies on the Mozilla XULRunner Framework, making it difficult to develop OpenCell further.

- New OpenCell to be developed using Qt/C++.

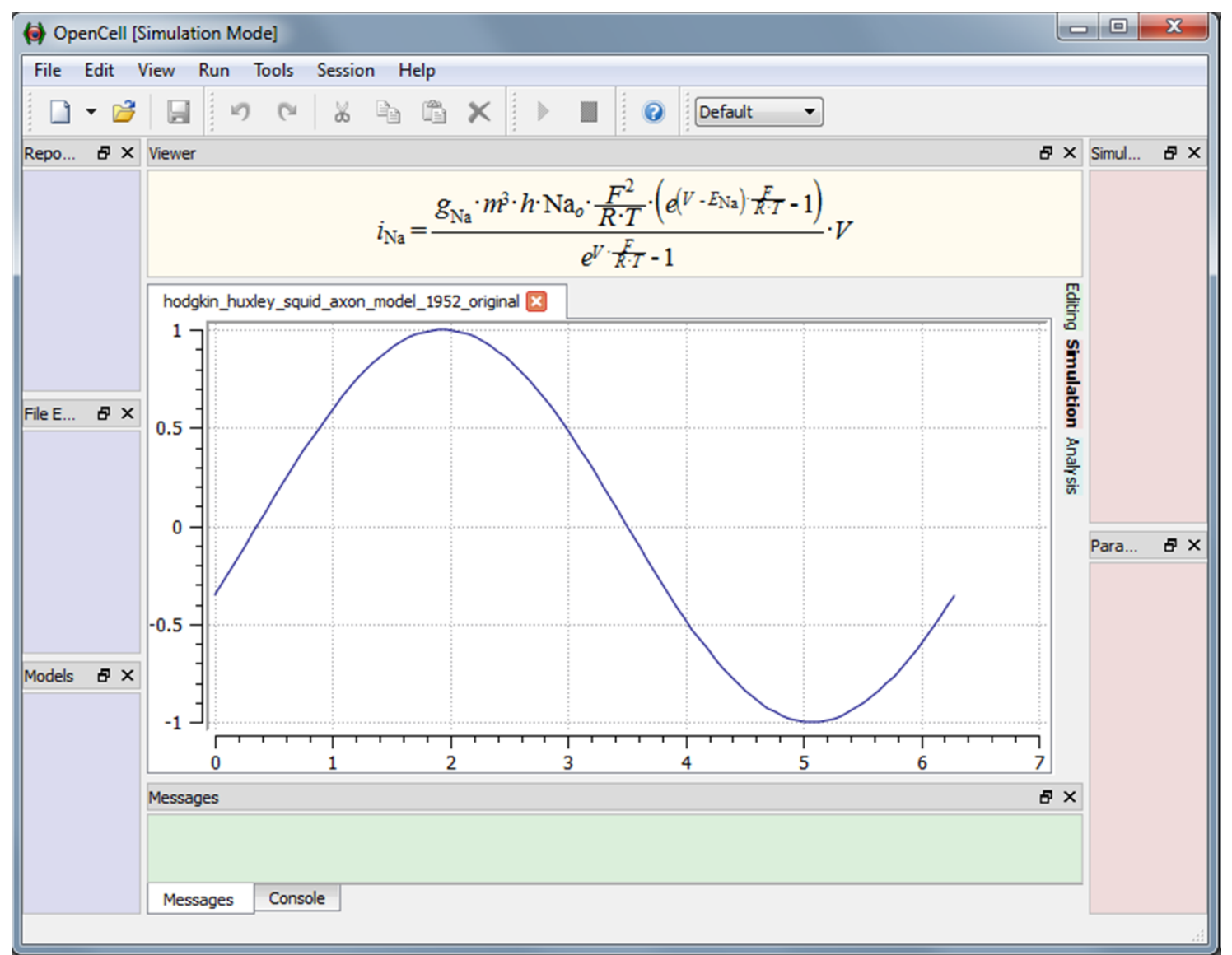




\section{Plan for a New OpenCell}

- OpenCell currently relies on the Mozilla XULRunner Framework, making it difficult to develop OpenCell further.

- New OpenCell to be developed using Qt/C++.

- Aiming at a working version by the end of next year.

- Initial focus will be on combining the current versions of OpenCell and COR.

- Next, the focus will be on metadata, ontologies, etc. 


\section{CONCLUSION}

- OpenCell 0.8 is soon to be released (OpenCell 0.8RC1 is currently available for download).

- There might be an OpenCell 0.9 (with the COR-like view).

- OpenCell, as we know it, is soon to enter maintenance mode.

- A new OpenCell is to be developed (led by Oxford), using the existing CellML 1.0/1.1 API (led by Auckland).

- A first public release is expected by the end of next year.

\section{www.opencell.org}

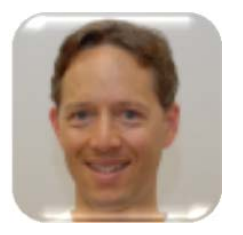

Randal Britten

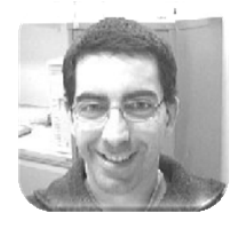

Alan Garny

r.britten@auckland.ac.nz alan.garny@dpag.ox.ac.uk j.marsh@auckland.ac.nz

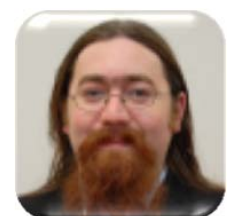

Justin Marsh

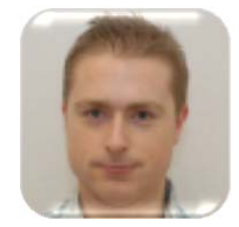

Andrew Miller a.miller@auckland.ac.nz 


\section{ACKNOWLEDGEMENTS}
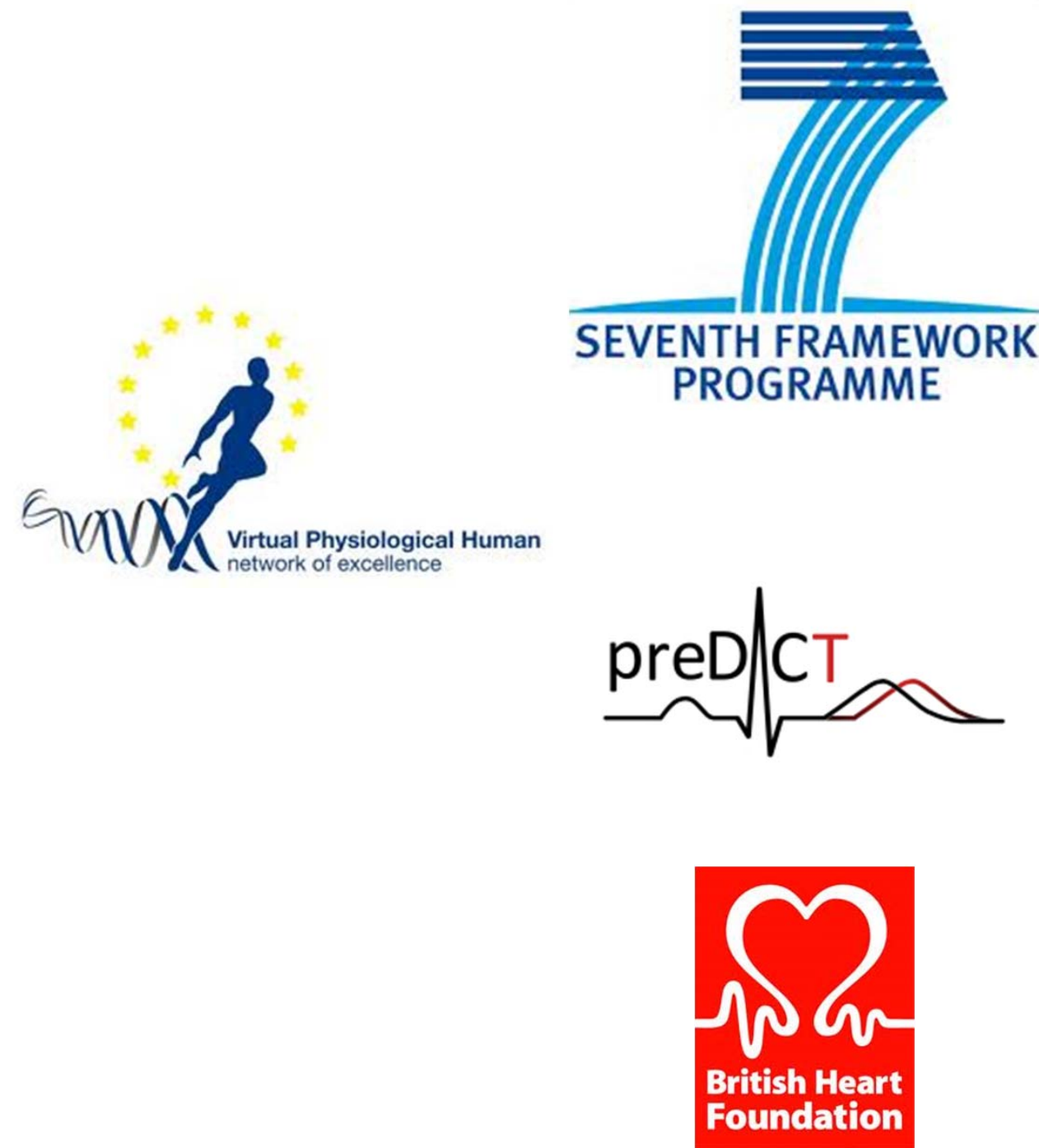